Cahiers de recherches médiévales

\title{
Illustrations et interprétations dans un manuscrit de l'Ovide moralisé (Arsenal 5069)
}

\section{Renate Blumenfeld-Kosinski}

\section{(2) OpenEdition \\ 12 Journals}

Édition électronique

URL : https://journals.openedition.org/crm/56

DOI : $10.4000 / \mathrm{crm} .56$

ISSN : 1955-2424

Éditeur

Honoré Champion

Édition imprimée

Date de publication : 10 décembre 2002

ISSN : 1272-9752

Référence électronique

Renate Blumenfeld-Kosinski, «Illustrations et interprétations dans un manuscrit de l'Ovide moralisé (Arsenal 5069) », Cahiers de recherches médiévales [En ligne], 9 | 2002, mis en ligne le 22 novembre 2006, consulté le 15 décembre 2022. URL : http://journals.openedition.org/crm/56 ; DOI : https:// doi.org/10.4000/crm.56

Ce document a été généré automatiquement le 15 décembre 2022.

Tous droits réservés 


\title{
Illustrations et interprétations dans un manuscrit de l'Ovide moralisé (Arsenal 5069)
}

\author{
Renate Blumenfeld-Kosinski
}

1 Les manuscrits illustrés posent la question du rapport entre texte et image d'une autre manière que les livres imprimés, car ces premiers n'offrent presque jamais le même programme d'illustration dans des exemplaires différents. Par conséquent chaque groupe d'illustrations nous offre une lecture différente du texte, une glose ${ }^{1}$, qui peut orienter notre interprétation. Dans certains cas les enluminures reproduisent d'une manière assez fidèle les événements décrits dans le texte, tels bon nombre de manuscrits des Grandes chroniques ou de la Bible ${ }^{2}$. Dans d'autres cas, une enluminure peut résumer ou exprimer d'une manière différente quelques idées abstraites ou l'idéologie sous-jacente d'un texte ${ }^{3}$.

2 Les manuscrits illustrés de l'Ovide moralisé se divisent en deux catégories qui correspondent plus ou moins aux deux possibilités indiquées ci-dessus. Dans un groupe les enluminures reproduisent des scènes mythologiques ou un aspect de l'interprétation allégorique ${ }^{4}$; dans l'autre chacun des quinze livres est orné d'un frontispice montrant un dieu ou une déesse païens sans que le rapport de cette figure au texte de ce livre soit évident. ${ }^{5}$ Cette tradition iconographiqe des imagines deorum remonte à Bersuire, Pétrarque et Boccace et ne se trouve pas dans les premiers manuscrits de l'Ovide moralisé6. En fait, un lecteur qui feuillette un manuscrit de ce deuxième groupe et ne voit que les grandes images des dieux antiques ne se rendrait pas compte que le texte consacre à peu près vingt-huit mille vers (d'un total de soixante-douze mille) à des allégories et des interprétations chrétiennes des fables d'Ovide.

3 Le premier groupe de manuscrits de l'ovide moralisé (Rouen, Bibliothèque municipale MS 0.4, 1315-25, 453 miniatures $^{7}$; Paris, Bibliothèque de l'Arsenal MS 5069, 1325-50, 302 miniatures ; Lyon, Bibliothèque municipale MS 742, env. 1390, 53 miniatures) comporte des miniatures illustrant les fables ovidiennes aussi bien que les interprétations 
chrétiennes. Mais même à l'intérieur de ce premier groupe une différentiation fort intéressante s'amorce à partir des illustrations ${ }^{8}$ : un mouvement vers le niveau littéral, signalé par le nombre de plus en plus réduit des enluminures ayant un rapport à l'interprétation allégorique. Tandis que le manuscrit de Rouen consacre $20 \%$ de ses illustrations au niveau allégorique, le manuscrit Arsenal 5069 ne propose que 10\% de ses images dans cette catégorie; dans le manuscrit de Lyon, qui appartenait à Jean, duc de Berry, seulement deux des images ont un rapport à la religion plutôt qu'à Ovide : la création du monde et la tour de Babel. Ici le Nouveau Testament s'efface complètement au profit de deux images célèbres de l'Ancien. Un autre manuscrit un peu plus tardif, BnF fr. 871 (env. 1400), dramatise la coexistence des domaines mythologique et religieux par le frontispice sur la création du monde. Il consacre un de ses quadrants à Prométhée qui, par son feu, anime une figure humaine sans vie, tandis que les trois autres montrent Dieu créant l'univers, les animaux et Eve. Ce folio fonctionne comme une alerte pour le lecteur qui doit s'attendre à cette juxtaposition des mondes antique et biblique et illustre donc l'idéologie sous-jacente à ce texte compliqué. Les mythes païens et les faits de la foi chrétienne font partie d'un système typologique où le mythe préfigure des événements et des idées des Nouveau et Ancien Testaments aussi bien que des préceptes moraux d'une valeur éternelle.

Quel est donc le rapport entre texte et image dans de tels manuscrits? Dans chaque cas quelqu'un a décidé de l'orientation du programme d'illustration. Les historiens de l'art sont de plus en plus d'accord que ce "quelqu'un» était probablement un clerc éduqué qui lisait le texte et qui, peut-être en consultation avec le patron ou l'artiste, fit le plan pour l'illustration d'un manuscrit donné9. Sur l'exemple du manuscrit Arsenal 5069 cet article montrera comment les enluminures d'un programme spécifique nous mènent à un niveau d'interprétation supplémentaire qui constitue une lecture particulière de ce texte complexe. C'est-à-dire que les images suppléent souvent à des lacunes - ou un manque de lien logique - dans les interprétations, en mettant en lumière des éléments iconographiques qui n'ont pas nécessairement de base textuelle.

5 L'Ovide moralisé, composé entre 1316 et 1328, est une vaste compilation et traduction de textes ovidiens, surtout des Métamorphoses mais aussi des Héroïdes et de textes vernaculaires comme le Roman de Troie de Benoît de Sainte-Maure ${ }^{10}$. Dans un rythme qui varie au cours des quinze livres, l'auteur anonyme (probablement un frère mineur) juxtapose des traductions assez fidèles du latin à des passages interprétatifs qui tirent une signification chrétienne (morale et biblique) du texte païen. La terminologie herméneutique est assez traditionnelle, utilisant des termes comme allégorie, exposition, fable, ou estoire, mais la mécanique interprétative est d'une complexité inouï $^{11}$ : des personnages ovidiens, voire des fables entières, sont soumises à des interprétations multiples, parfois hiérarchisées et qui ne sont pas toujours évidentes.

Dans certains cas une équation est établie qui relève d'un élément narratif : Orphée est le Christ parce qu'il est descendu, comme Lui, dans les enfers; ou c'est peut-être un objet qui à partir de sa valeur symbolique se transforme: tel le bateau d'Enée qui comme «nef de l'Église » joue un rôle important dans une allégorie prolixe au Livre 14. Les possibilités interprétatives sont sans limites. Ce qui nous intéresse dans le contexte des enluminures c'est dans quelle mesure les images ajoutent un sens supplémentaire, ou établissent des liens entre le texte ovidien et son interprétation chrétienne qui auraient échappé au lecteur sans l'aide visuelle. 
7 Une étude du choix particulier des illustrations nous offre la possibilité d'une compréhension plus approfondie d'un mode de pensée fondamental dans la culture médiévale: la pensée typologique. Mais elle nous montrera aussi que la typologie comportait une certaine flexibilité, car un artiste et son conseiller disposaient d'une grande liberté de choix. Notre étude porte sur le manuscrit 5069 de la Bibliothèque de l'Arsenal, le deuxième dans la famille la plus ancienne. Simplifiées par rapport aux illustrations du manuscrit de Rouen, les enluminures ne sont pas très sophistiquées du point de vue artistique ${ }^{12}$. Il s'agit donc d'un manuscrit «moyen » qui par là peut servir à titre d'exemple.

Un lecteur médiéval se trouve donc en face d'un mélange chrétien/païen d'illustrations qui met en évidence les pratiques interprétatives de l'ovide moralisé mais qui a aussi un côté déconcertant. Par exemple, le folio $27^{\mathrm{r}}$ du manuscrit Arsenal 5069 nous montre Jupiter en train de kidnapper Europe [fig.1], tandis que le verso comporte une image du Christ qui porte la croix [fig. 2]. Quelle est la relation possible entre ces deux scènes? La notion du sacrifice et du 'bœuf' se trouve au centre de cette interprétation. Le texte nous dit que le Christ était «aussi come buef que l'on maine/ Au sacrefice souffrir mort» (2.5128-5129). Et comme Jupiter s'était transformé en 'bœuf' pour enlever Europe, l'auteur de l'ovide moralisé nous offre l'équation Jupiter-bœuf-sacrifice païen-sacrifice chrétien=Christ. Sans base textuelle cette relation particulière nous échapperait. En tout cas, il n'y a que peu de ressemblance iconographique entre les deux images, sauf peut-être le port sur le dos d'une femme et de la croix. Un seul examen des images ne conduirait donc probablement pas le lecteur à saisir les rapports profonds existant entre le rapt d'Europe et le port de la croix.

9 Un cas beaucoup plus complexe se trouve aux folios $71^{\mathrm{v}}$ et $73^{\mathrm{r}}$. Deux images de gens qui prient ont des sens opposés. La première figure [fig. 3], priant devant une croix sur un autel, représente «clergie et philosophie »; elle fait partie de l'allégorie de la fontaine de Pégase dans le Livre 5 qui figure «la fontaine de clergie» pleine "dou boivre de philozophie » (5.2528sq.). Par contre, la figure sur le folio $73^{\mathrm{r}}$ [fig.4] adore une idole. Il faut souligner ici qu'en dépit du motif païen cette image ne vient pas du texte ovidien mais appartient au domaine interprétatif, celui de l'histoire du géant Typhée (5.1814-1832) qui avait chassé les dieux païens de l'Égypte (plus tard il sera interprété comme le Christ [5.2908]), où l'adoration des idoles avait son origine (5.2736-2739), un fait déjà attesté par Fulgence. La poésie, selon l'auteur de l'Ovide moralisé, était un moyen puissant dans cette adoration des faux dieux (5.2752). Ici le programme iconographique nous permet de percevoir une distinction importante, mais pas vraiment soulignée dans le texte qui, par son entassement d'interprétations, n'en privilégie aucune. Les images tirent une leçon importante du texte: un même geste peut cacher des obéissances contraires, à la vraie foi ou à l'idolâtrie. La poésie, médiatisée par la "clergie», est liée à la foi, la poésie sans «clergie " mène à l'idolâtrie. Ces images comportent donc une mise en garde : ne pas se laisser séduire par les seuls gestes d'adoration (car ils sont identiques, qu'il s'agisse des fidèles et des infidèles) et bien 'supplémenter' la poésie par la «clergie», pour ne pas aboutir aux portes de l'enfer. Cette leçon pour les poètes est mise en évidence beaucoup plus clairement par les images que par le texte. Par contre, l'équation entre le géant et le Christ dans ce passage qui, pour nous lecteurs modernes, est peut-être plus importante, n'a pas attiré l'attention de l'artiste ; il n'y en a aucune illustration. 
10 «Clergie » et philosophie marquent la vie contemplative, un idéal proposé maintes fois dans notre texte, notamment dans le jugement de Pâris (Livre 11). Dans la tradition interprétative remontant à Fulgence, les trois déesses qui se présentent à Pâris signifient les vies active (Junon), voluptueuse (Vénus), et contemplative (Pallas). Le manuscrit Rouen 0.4 , le plus ancien, montre, dans trois compartiments, une femme à la quenouille, une femme qui se peigne en tenant un miroir et, à droite, une nonne se penchant sur un livre ouvert ${ }^{13}$. Selon Fulgence, la vie contemplative est incarnée surtout par les évêques, les prêtres et les moines ${ }^{14}$. On a l'impression que l'illustrateur $\mathrm{du}$ manuscrit de Rouen voulait combiner la nature féminine des déesses avec le précepte fulgentien, ce qui l'a mené à l'image d'une nonne. Dans notre manuscrit, le groupe a un air moins cohérent, car à côté de deux femmes qui s'adonnent aux mêmes activités que celles figurées dans le manuscrit de Rouen, voilà un moine barbu à l'air d'ermite absorbé par sa lecture [fol. 153 v , fig. 5]. Le texte fulgentien l'emporte ici sur la conformité de sexe pour les trois vies. Notre illustrateur semble vouloir exclure les femmes de cette vie qui, dans l'histoire de Marthe et Marie, est jugée par le Christ comme «la bonne part » (Luc 10.38-42) $)^{15}$.

11 Mais les clercs n'attirent pas toujours la bienveillance de notre artiste. Par exemple, dans le Livre 5, Phinée, l'oncle d'Andromède, s'attaque à Persée, et par conséquent est interprété comme l'ennemi du Christ. Dans le texte il y a une quantité d'interprétations qui s'appuient sur des événements de la vie de Jésus, mais le choix de l'artiste est tombé sur l'interprétation mise en lumière par la rubrique «Ci devise l'exposicion de Phineus qui volt occire Perseus et comment il est comparé a ceuls qui veulent corrompre les mariages ». Phineus représente ceux qui ont choisi «les mondains delis,/ Et pour l'aise de la char faire/ Veulent a Dieu l'ame soustraire » (5.129-131). Le mariage corrompu est celui de Dieu et de l'âme (5.161-162). Pour notre artiste, les mondains sont ceux qui s'intéressent aux femmes malgré leur vocation cléricale: les clercs corrompus qui embrassent les femmes comme le fait l'homme sur le folio $64^{\mathrm{v}}$ [fig. 6 ${ }^{16}$. Mais - et c'est le point crucial-, les clercs ne sont pas visés explicitement dans le texte. C'est exclusivement par l'illustration que la critique des «mondains » s'inscrit dans toute une série d'accusations contre un clergé corrompu et des moines pervertis qu'on trouve ailleurs dans le texte.

Ce clerc lascif nous mène au domaine de la transgression sexuelle, thème ovidien par excellence. Des personnages comme Iphis, Byblis, ou Pasiphaé sont intégrés dans un système interprétatif complexe ${ }^{17}$. Dans le Livre 9 , nous trouvons l'histoire d'Iphis, la jeune fille élevée en garçon par sa mère afin de la protéger des dessins homicides de son père qui avait désiré un fils. Elle tombe amoureuse d'une jeune fille qu'elle épouse après une tranformation en homme à la dernière minute. L'auteur de l'Ovide moralisé nous offre comme toujours une multiplicité d'interprétations. Aux niveaux littéral et moral, Iphis est une femme qui, à l'aide d'« un membre attaché », essaie de séduire une jeune fille. La rubrique nous informe là-dessus : «Ci dist la moralité de la fable de celle qui se fist homme pour avoir une pucelle pour faire en sa volonté » (fol. 131 r). Sur le même folio, l'image nous montre l'étreinte amoureuse de deux femmes [fig. 7]. L'illustrateur esquive donc l'interprétation salvatrice qui voit dans Iphis l'âme pècheresse sauvée par sa mère Aréthusa (= l’ Église).

13 Par contre, pour Byblis, dévorée d'un désir incestueux pour son frère Caunus et métamorphosée en fontaine, l'artiste privilégie l'interprétation spirituelle qui la représente comme «divine sapience» (Livre 9.2586), illustrée par le Gnadenstuhl (fol. 
$\left.129^{v}\right)$. Le niveau littéral ou historique indique que Byblis est une prostituée qui se donne " comme une fontaine» à tout le monde. À partir de la seule illustration, le lecteur ne pourrait jamais deviner qu'il s'agit ici d'un autre récit de transgression sexuelle. Pour Byblis, l'inceste disparait donc du premier plan, tandis que, pour Iphis, l'amour lesbien, condamné ici comme perversion, est mis en lumière par l'illustration.

Le Livre 4 nous offre un autre exemple de la manière dont un élément iconographique peut établir un lien entre récit ovidien et interprétation chrétienne peu visible, ou même invisible, dans le texte. Sur le folio $43^{\mathrm{r}}$ une scène dramatique met en évidence le martyre par l'épée de plusieurs personnes [fig. 8]. Dans le texte, cette scène est liée à l'histoire de Pyramus et Thisbé par le motif de la mûre dont le jus évoque vaguement le sang du Christ $(4.1190)^{18}$. Le poète nous encourage à souffrir pour le Christ comme l'avaient fait les martyrs. Mais l'énumération des moyens de martyre (« enmurer, tenir en chartre, lapidiez, batus, blastengiez, tempté, mort »-Livre 4.1208sq.) ne mentionne pas une épée. Le meurtre par l'épée, qui donne une force extraordinaire à cette image, est donc une invention de l'illustrateur qui établit ainsi un lien absent du texte: les suicides de Pyramus et de Thisbé par une épée (fols. $42^{\mathrm{r}}$ et $42^{\mathrm{v}}$ ) sont mis en relation avec les martyrs. Cette équation entre les deux amants et les martyrs n'apparaît pas dans le texte où ni Pyramus ni Thisbé ne sont interprétés (seul le lion est comparé au diable). Ici l'illustration fournit une interprétation supplémentaire qui donne le rôle de martyr aux deux jeunes amoureux.

Parfois une illustration surgit du texte par une allusion presque invisible au lecteur. Pourquoi, par exemple, l'Annonce faite à Marie - jamais mentionnée dans le texte apparait-elle comme illustration au milieu de la fable de Céphalus et Procris? Il est possible que les lignes sur le désir du Christ de se faire homme en descendant dans le "ventre à la vierge pucelle,/ Qui est temple et divine celle " (7.3447sq.) aient suggéré à l'illustrateur - ou à son clerc conseiller - l'Annonciation, le prélude à l'Incarnation du Christ. En tout cas, cette illustration, absente des autres manuscrits de ce groupe, reflète une lecture particulière qui donne une orientation individuelle à ce manuscrit.

Les deux sœurs Ariane et Phèdre se trouvent au centre de notre dernier exemple. Ovide nous raconte comment Thésée, après sa victoire sur le Minotaure, avait enlevé les deux jeunes femmes, mais avait abandonné Ariane qui s'était endormie dans le bateau [fol. $111^{r}$, fig. 9]. Le motif du sommeil engendre l'allégorie de l'Église et de la synagogue : comme Ariane endormie laissa partir Thésée, la synagogue ne retint pas le Christ qui s'enfuit avec l'autre sœur = l'Église [fol. 111v, fig. 10]. Le parallèle entre le texte et cette interprétation réside donc dans le motif d'une femme aux yeux fermés et d'une autre aux yeux ouverts. L'iconographie la plus commune de la synagogue depuis le XII ${ }^{\mathrm{e}}$ siècle était l'image d'une femme aux yeux bandés dont la couronne glisse, comme l'annonce Jérémie dans ses Lamentations $5.16-17^{19}$. Mais notre artiste nous montre une synagogue aux yeux fermés : elle est « endormie » comme Ariane. L'illustration s'écarte donc de la tradition iconographique prévalente afin d'établir un lien plus clair entre le récit ovidien et l'interprétation allégorique.

17 Chaque manuscrit raconte sa propre histoire. Il est remarquable que seul un tiers des trente illustrations ayant un rapport au niveau allégorique ou moral du manuscrit Arsenal 5069 soit identique à celles de Rouen 0.4 (qui en compte quatre-vingt-dix), l'ancêtre du manuscrit de l'Arsenal. Les manuscrits plus tardifs s'éloignent de plus en plus de ces modèles et leurs enluminures montrent presque exclusivement des scènes ovidiennes. L'autre famille iconographique, mentionnée au début de cet article, choisit 
les imagines deorum au lieu des images narratives ${ }^{20}$. Cette absence croissante des images allégoriques et religieuses suggère une transformation dans la lecture de l'ovide moralisé $^{21}$. Ce qui compte de plus en plus, semble-t-il, est la traduction française des Métamorphoses et d'autres textes latins plutôt que la christianisation de ces textes. En feuilletant les manuscrits, un lecteur ne rencontre plus les images du Christ, des martyrs, ou de l'Église personnifiée, mais la vie fourmillante des contes ovidiens ou la glorification des dieux païens. Cette réorientation de la lecture reflète sans doute un mouvement vers le pré-humanisme, pourtant peu visible dans les passages textuels. C'est au moyen des illustrations que des générations d'artistes mettent en lumière cette transformation.

\section{ANNEXES}

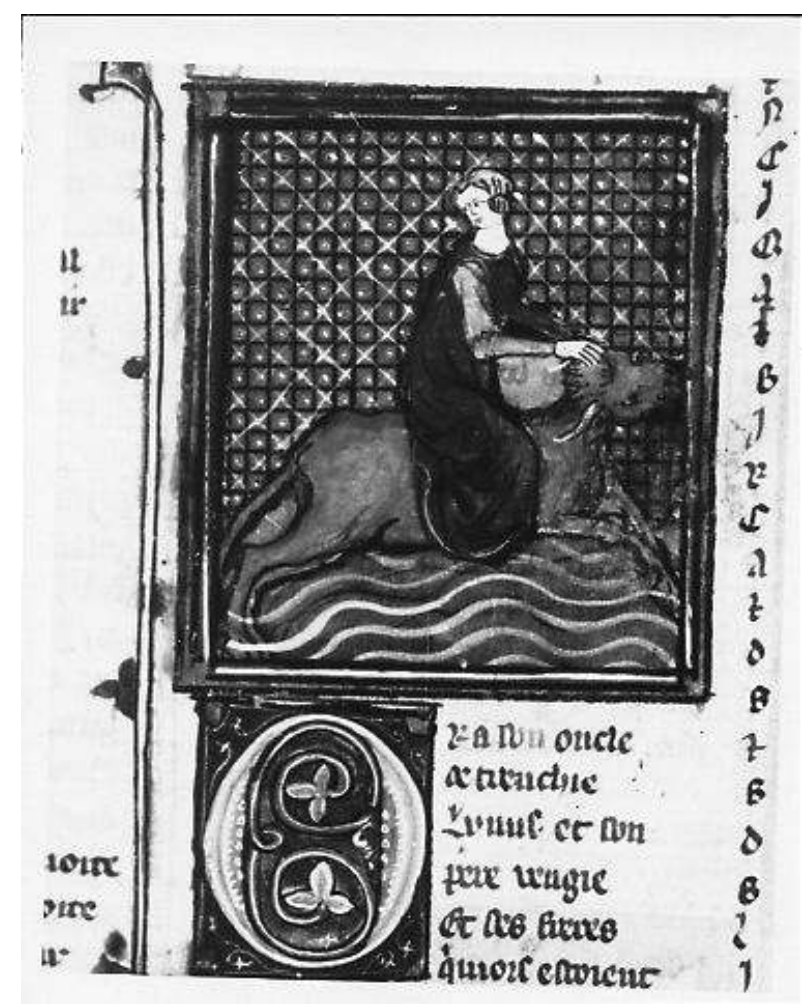

Fig.1 MS Arsenal 5069, fol. 27 , Jupiter en forme de boeuf emporte Europe. Bibliothèque nationale de France 


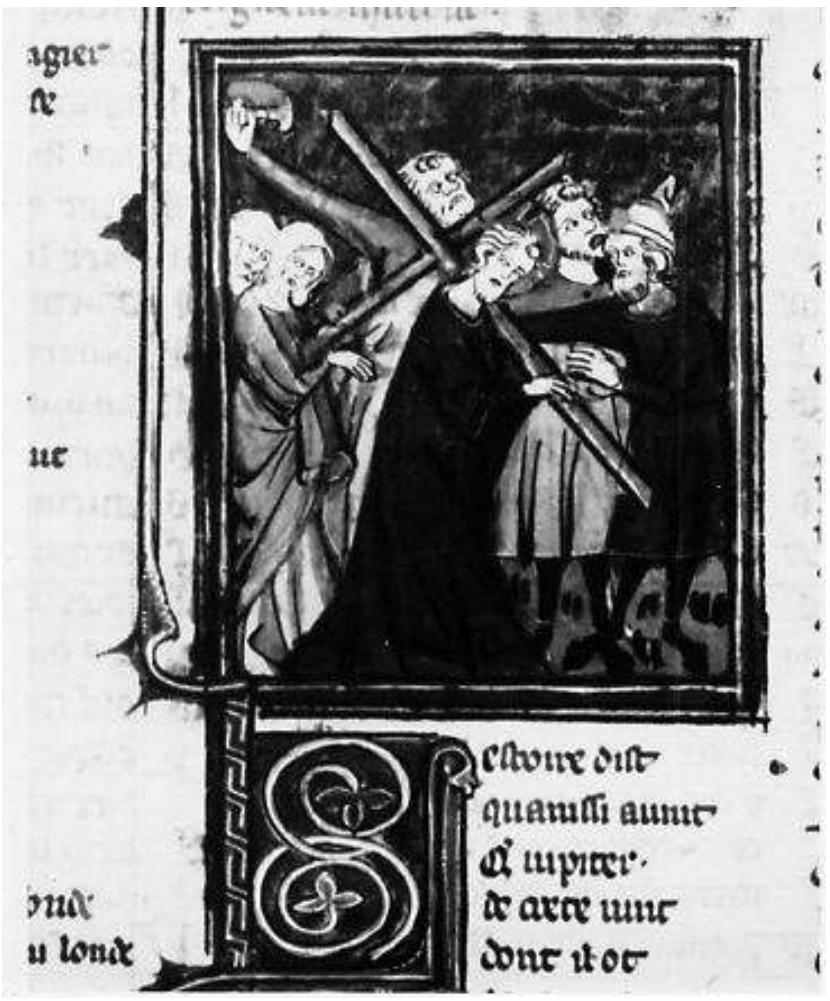

Fig. 2 MS Arsenal 5069, fol. 27v , le Christ portant la croix Bibliothèque nationale de France

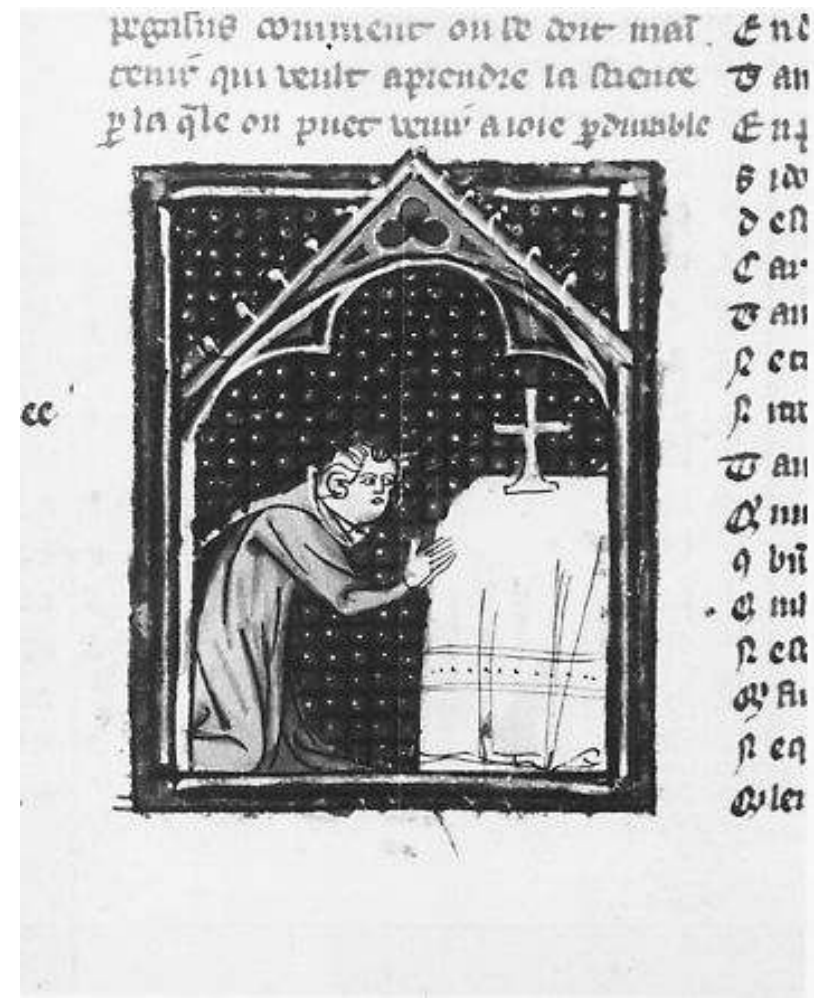

Fig. 3 MS Arsenal 5069, fol. $71^{v}$, prière devant un autel. Bibliothèque nationale de France 


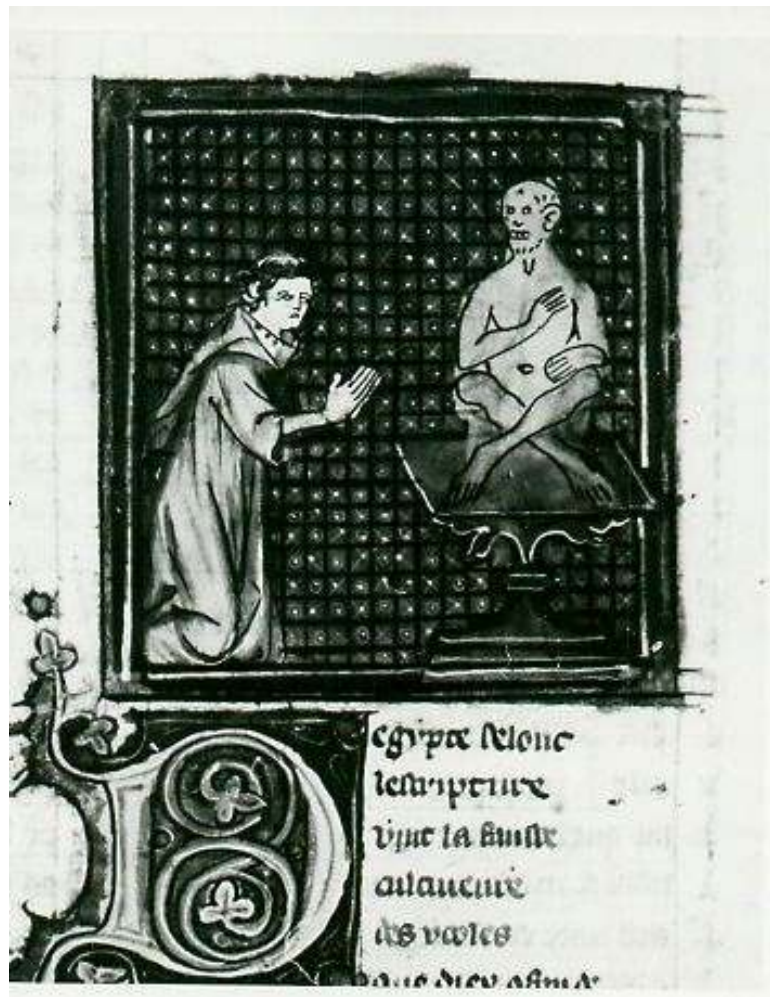

Fig. 4 MS Arsenal 5069, fol. $73^{\mathrm{r}}$, prière devant une idole. Bibliothèque nationale de France

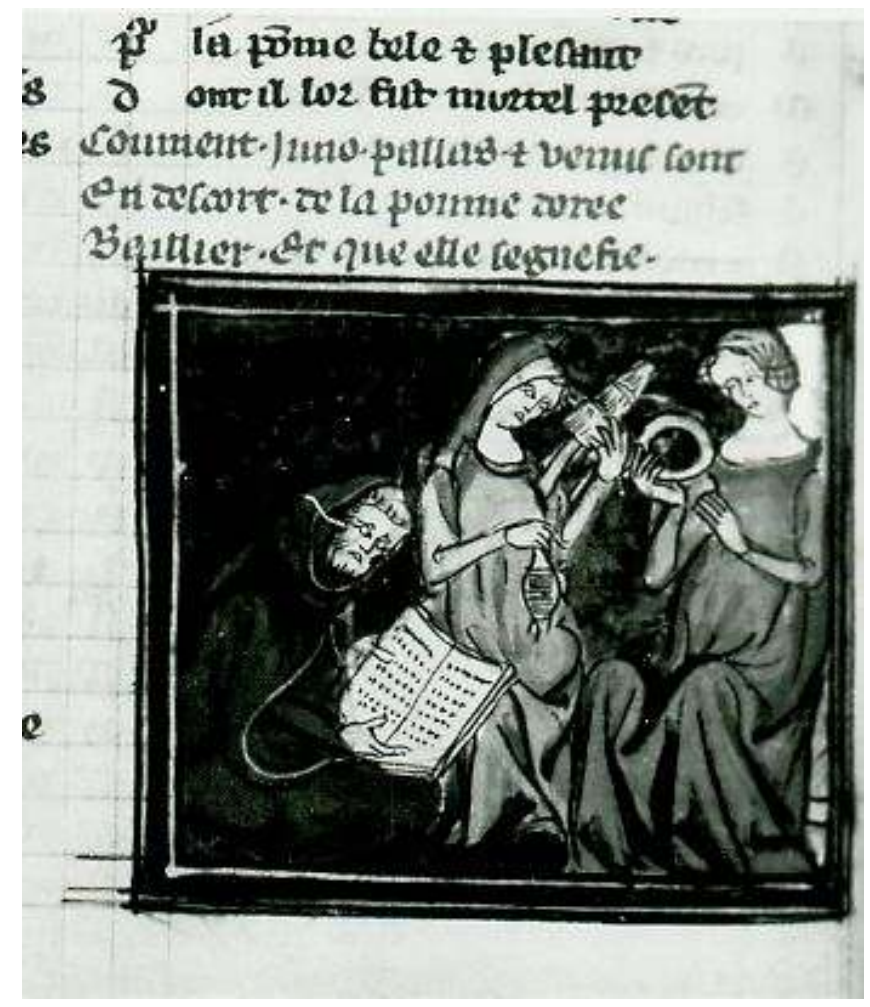

Fig. 5 MS Arsenal 5069, fol. $153^{v}$, les vies comtemplative, active, et voluptueuse. Bibliothèque nationale de France 


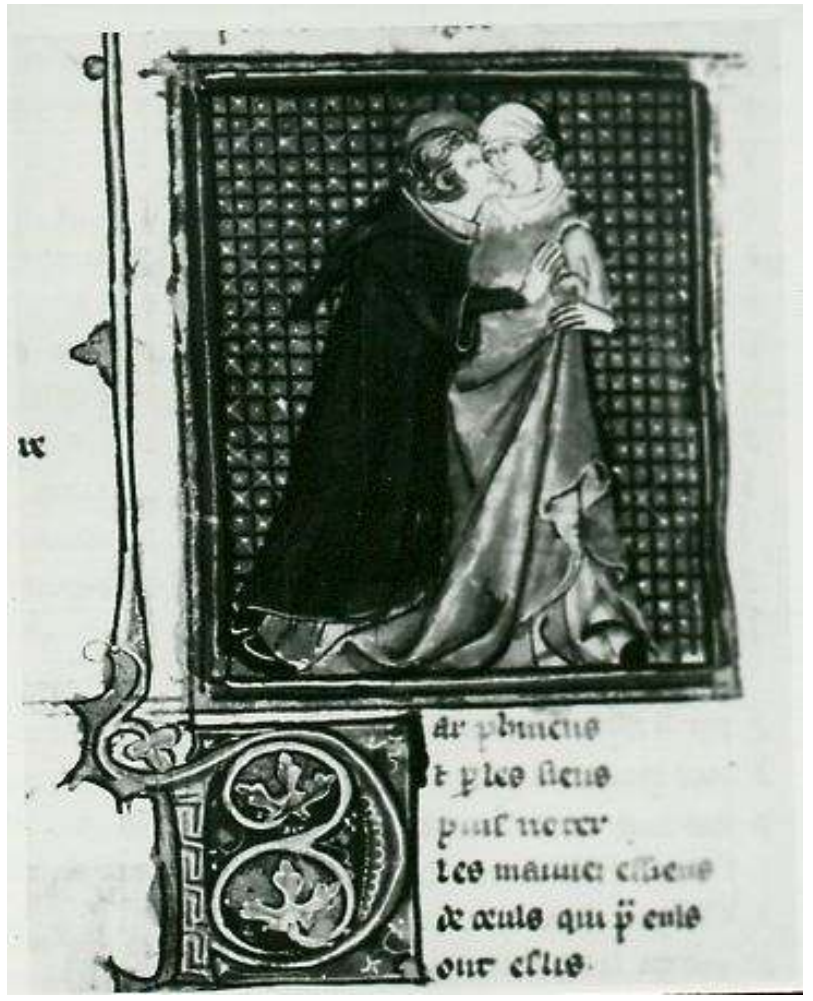

Fig. 6 MS Arsenal 5069, fol. $64^{\mathrm{v}}$, homme embrassant une femme. Bibliothèque nationale de France

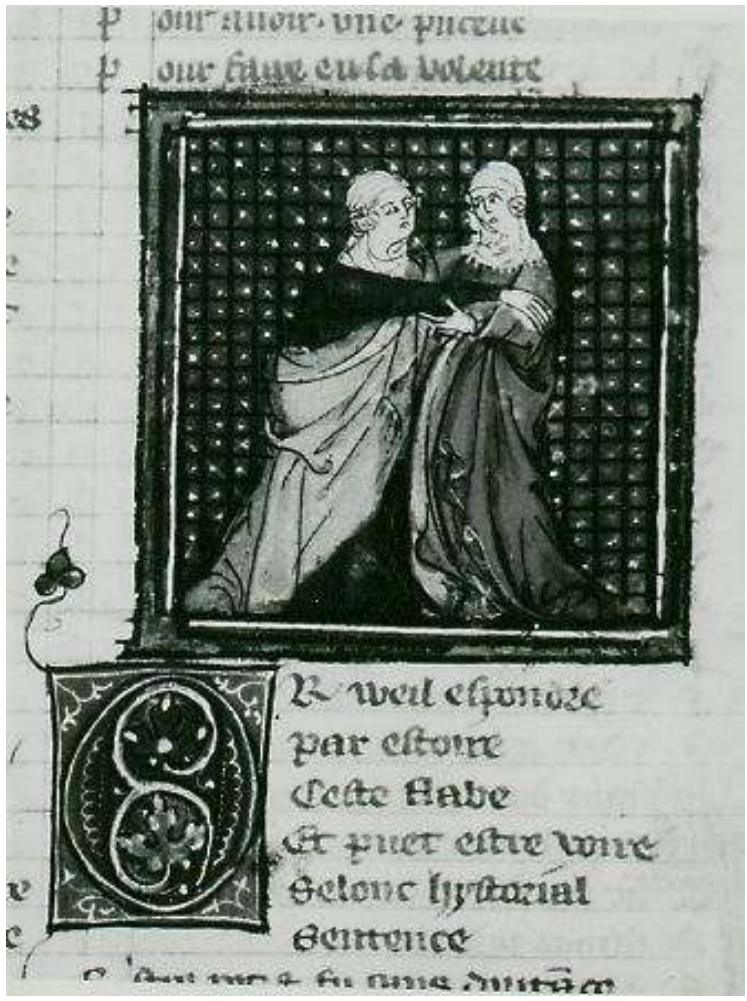

Fig. 7 MS Arsenal 5069, fol. $131^{\mathrm{r}}$, étreinte amoureuse de deux femmes. Bibliothèque nationale de France 


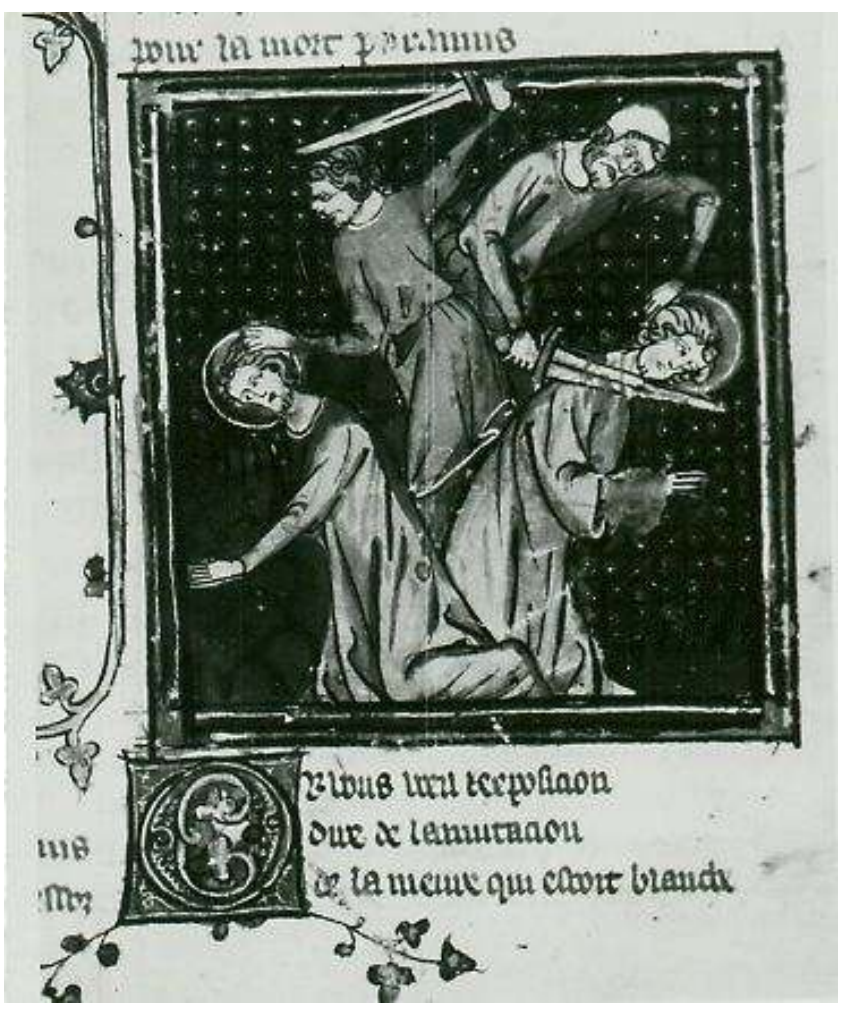

Fig. 8 MS Arsenal 5069, fol. $43^{\mathrm{r}}$, martyre par l'épée. Bibliothèque nationale de France

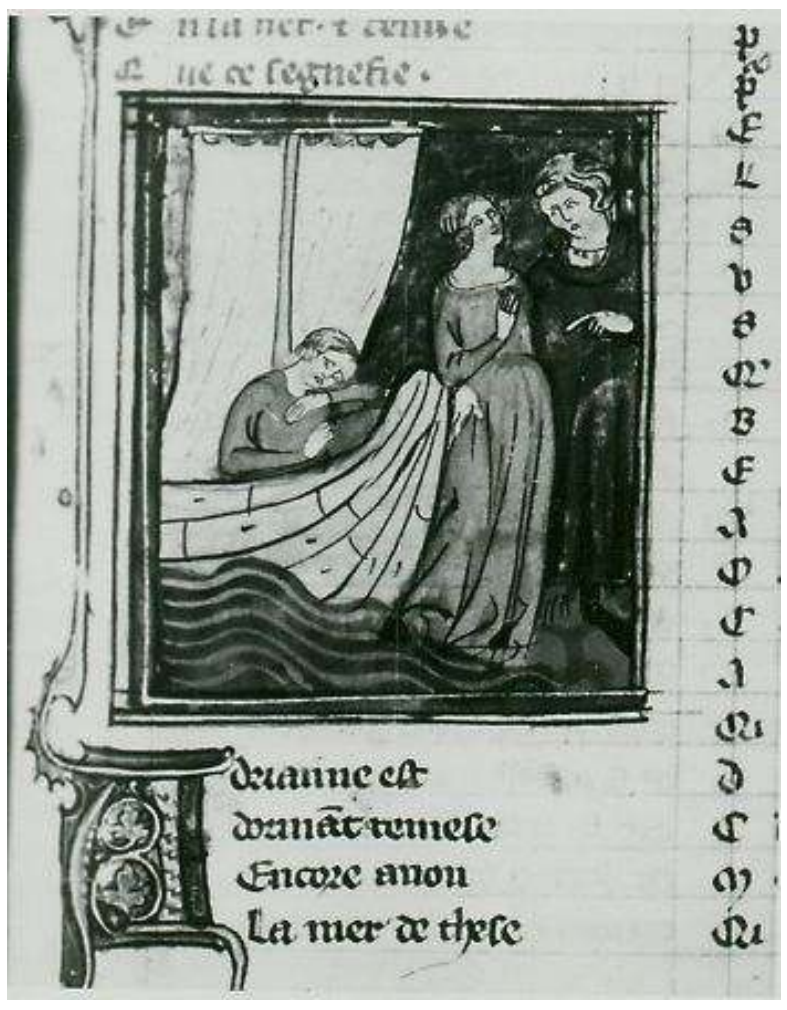

Fig. 9 MS Arsenal 5069, fol. $111^{\mathrm{r}}$, Ariane endormie dans le bateau. Bibliothèque nationale de France 


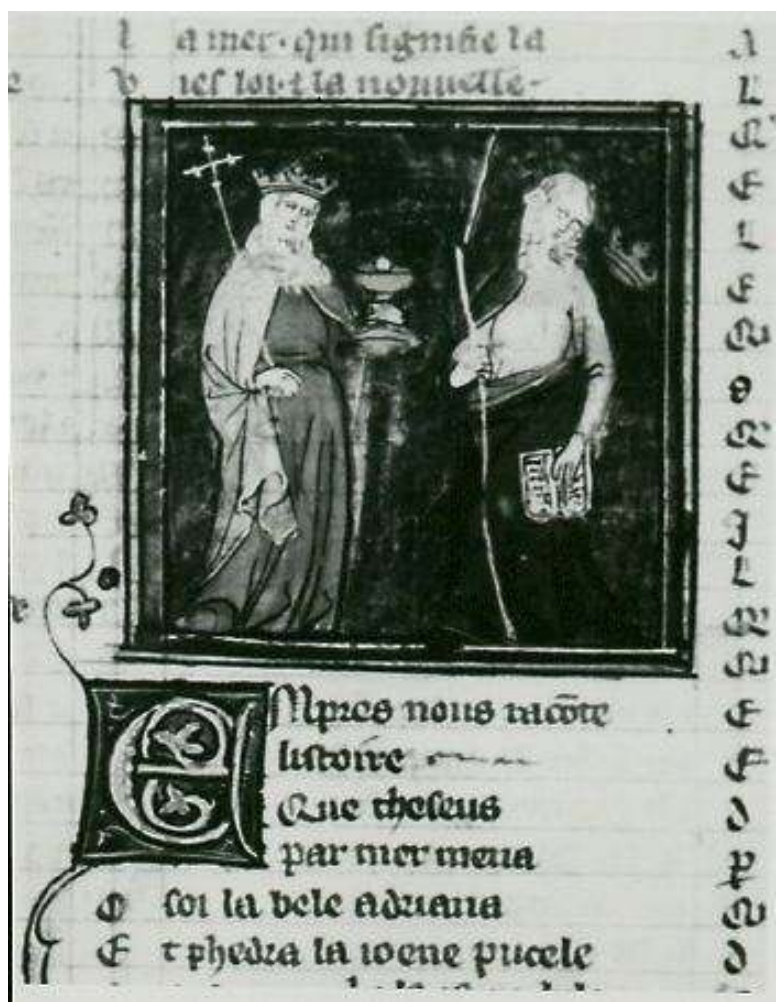

Fig. 10 MS Arsenal 5069, fol. 111v , Église et Synagogue aux yeux fermés; sa couronne tombe. Bibliothèque nationale de France

\section{NOTES}

1.S. Huot, The Romance of the Rose and its Medieval Readers. Interpretation, Reception, Manuscript Transmission, Cambridge, Cambridge University Press, 1993, chap. 8.

2.A. D. Hedeman, The Royal Image. Illustrations of the Grandes Chroniques de France, 1274-1422, Berkeley, University of California Press, 1991. S. L. Hindman, "The Role of Author and Artist in the Procedure of Illustrating Late Medieval Texts ", Text and Image. Acta vol. 10, 1983, pp. 27-28.

3.S. Hindman montre, par exemple, comment le frontispice d'un manuscrit de la Chronique abrégée de Guillaume de Nangis fournit une interprétation supplémentaire en représentant une allégorie compliquée de la Fleur-de-Lis qui n'apparaît pas dans le texte lui-même. Hindman, art. cit., p. 32 et fig. 4.

4.On trouve aussi des exemples où des métaphores sont traduites en des images narratives : dans une illustration d'un manuscrit d'une traduction française de $L a$ Consolation de philosophie de Boèce (MS 12, Trinity Hall, Cambridge) les ennemis de Paulinus (des «chiens » pour Boèce) sont représentés comme des chiens sauvages dont Boèce protège son ami. Voir R. A. Dwyer, Boethian Fictions. Narratives in the Medieval French Versions of the Consolatio Philosophiae, Cambridge, Mass., Medieval Academy of America, 1976, p. 28 et fig. 1.

5.On trouve pourtant quelques liens entre le frontispice et le texte dans les manuscrits figurant les imagines deorum. Dans BnF fr. 373, par exemple, le Livre 4, qui contient l'histoire de Mars et Vénus, est précédé de l'image de Vulcain, tandis que le Livre 9 commence par l'image d'Hercule qui y apparaît. Mais il n'y aucune explication des 
images ni des attributs des figures. Pour une orientation générale voir E. Panofsky et $\mathrm{F}$. Saxl, «Classical Mythology in Medieval Art », Metropolitan Museum Studies, 4 (1932-33), pp. 228-280. Panofsky, Renaissance and Renascence in Western Art, Stockholm, 1960, réimpr. New York, Harper and Row, 1972. J. Seznec, La Survivance des dieux antiques, Studies of the Warburg Institute, vol. XI, 1940.

6.Il est important de rappeler que des manuscrits appartenant à une même famille textuelle et ayant des dates presque identiques peuvent avoir des programmes d'illustration complètement différents. Pour une liste des manuscrits groupés par famille voir M.-R. Jung, « Aspects de l'Ovide moralisé », Ovidius redivius. Von Ovid zu Dante, éd. M. Picone et B. Zimmermann, Stuttgart, M\&P, 1994, pp. 170-171.

7.Sur ce manuscrit voir J. Porcher, Les manuscrits à peintures en France du XIII ${ }^{e}$ au XVI siècle, Paris, Bibliothèque Nationale, 1955, p. 26, no. 40.

8.C. Lord étudie ces trois manuscrits dans "Three Manuscripts of the Ovide moralisé ", Art Bulletin 57 (1975), pp. 161-175.

9.Hindman, «The Roles of Author and Artist », pp. 34-41.

10.Pour les sources voir P. Demats, Fabula. Trois études de mythographie antique et médiévale, Genève, Droz, 1973.

11.Sur tous les aspects de l'herméneutique de l'Ovide moralisé voir le chap. 3 de mon livre Reading Myth. Classical Mythology and Its Interpretations in Medieval French Literature, Stanford, Stanford University Press, 1997.

12.Lord parle d'un style international, produit hâtivement dans un grand atelier («Three Manuscripts, » p. 168). Ce manuscrit appartenait à Charles de Croy. Voir Jung, "Aspects de l'Ovide moralisé ", p. 171.

13.Fol. 286r . Voir fig. 7 dans M. Ehrhart, The Judgment of the Trojan Prince Paris in Medieval Literature, Philadelphia, University of Pennsylvania Press, 1987.

14.Voir Ehrhart, op. cit., pp. 23-27.

15.Il est possible que ce soit surtout l'influence de Fulgence qui détermine le choix du moine barbu. Car dans le Livre 6, lors de la transformation d'Antigone en cigogne, la vie contemplative est représentée par deux nonnes devant un autel (fol. $\left.82^{\mathrm{r}}\right)$.

16. Bien que l'homme ne soit pas tonsuré, l'apparence de cette figure (y compris ses vêtements) suggère qu'il s'agit d'un clerc plutôt que d'un laïc.

17.Sur Pasiphaé, voir mon article «The Scandal of Pasiphaé : Narration and Interpretation in the Ovide moralisé » Modern Philology 93, 1996, pp. 307-326. Sur Iphis et Byblis mon Reading Myth, p. 108sq., 120sq.

18. L'allégorie du récit de Pyramus et Thisbé est beaucoup plus courte (4.1170-1267) que le récit lui-même (4.219-1169), ce qui fait exception dans ce Livre 4. Elle est aussi une des plus vagues dans le texte entier.

19.Voir des exemples multiples dans W. S. Seifert, Synagogue and Church in the Middle Ages. Two Symbols in Art and Literature, New York, Frederick Ungar Publishing, 1970, chap. 9 «Synagoga's Blindfold.» Il y a aussi des exemples de la synagogue aveugle aux yeux fermés, mais la tradition des yeux bandés était plus répandue.

20.Dans la plupart des manuscrits de l'Ovide moralisé, les descriptions des imagines manquent. Ces descriptions viennent du Mythographe du Vatican III, de Bersuire, de Pétrarque et de Boccace. Voir Panofsky, op. cit. et Seznec, op. cit. et infra, l'art. de N. Koble.

21.R. Brown-Grant montre que Jean Miélot modifie le sens de l'Epistre d'Othéa de Christine de Pizan d'une manière similaire. Par des changements textuels mais surtout 
par les illustrations sa version s'écarte de la signification morale et allégorique donnée par Christine aux contes ovidiens et troyens au profit d'une lecture plus littérale. Voir Brown-Grant, «Illumination as Reception : Jean Miélot's Reworking of the 'Epistre d'Othea,' ", The City of Scholars : New Approaches to Christine de Pizan, ed. M. Zimmermann et Dina de Rentiis, Berlin, De Gruyter, 1994, pp. 260-271.

\section{AUTEUR}

RENATE BLUMENFELD-KOSINSKI

University of Pittsburgh 